Notre Dame Journal of Formal Logic

Volume 47, Number 4, 2006

\title{
Level Compactness
}

\author{
Gillman Payette and Blaine d'Entremont
}

\begin{abstract}
The concept of compactness is a necessary condition of any system that is going to call itself a finitary method of proof. However, it can also apply to predicates of sets of formulas in general and in that manner it can be used in relation to level functions, a flavor of measure functions. In what follows we will tie these concepts of measure and compactness together and expand some concepts which appear in the author's master's thesis, "Inference and Level." We will also provide some applications of the concept of level to the "preservationist" program of paraconsistent logic. We apply the finite level compactness theorem in this paper to get a Lindenbaum flavor extension lemma and a maximal "forcibility" theorem. Each of these is based on an abstract deductive system $X$ which satisfies minimal conditions of inference and has generalizations of 'and' and 'not' as logical words.
\end{abstract}

\section{Introduction}

The concept of compactness is a necessary condition of any system that is going to call itself a finitary method of proof. However, it can also apply to predicates of sets of formulas in general and in that guise it can be applied to level functions. Level functions are set functions akin to measure functions. In what follows we will tie measure and compactness together (via level) and expand some concepts which appear in the author's master's thesis, "Inference and Level." We will also provide some applications of level to paraconsistent logic. One such inference relation, in particular, is forcing, a la Schotch and Jennings [4] and [5].

\section{Measure to Level}

A measure is a function $\mu: \mathcal{B}(E) \longrightarrow \mathbb{R} \cup\{\infty\}$ defined on a $\sigma$-algebra $\mathscr{B}(E)$ over a set $E$.

Definition 2.1 A $\sigma$-algebra on $E$ is a collection of subsets of $E, \mathscr{B}(E)$ such that

Received May 12, 2006; accepted October 19, 2006; printed December 28, 2006

2000 Mathematics Subject Classification: Primary, 03B53; Secondary, 03B22, 28B10

Keywords: measure, level, paraconsistent logic, compactness, forcing

(c) 2006 University of Notre Dame 
1. $\varnothing \in \mathscr{B}(E)$,

2. any countable union of elements of $\mathscr{B}(E)$ is an element of $\mathscr{B}(E)$, and

3. the complement of any element of $\mathcal{B}(E)$ in $E$ is an element of $\mathcal{B}(E)$.

For any set $A$ the power set $\mathcal{P}(A)$ is a $\sigma$-algebra. ${ }^{1}$

Measure functions are required to have the following properties:

1. $\mu(A) \geq 0$ for $A \in \mathscr{B}(E)$, with equality if $A=\varnothing$;

2. $\mu\left(\bigcup_{i=0}^{\infty} A_{i}\right)=\sum_{i=0}^{\infty} \mu\left(A_{i}\right)$ for any sequence of disjoint sets $A_{i} \in \mathscr{B}(E)$ (Jao [3]).

The additive property above is a very strong condition and will not survive generalization to a notion that we shall call level. We will, however, maintain the convention that all sets will have at least level 0 , and the empty set will have level 0 .

Like measure, level is represented by a function called, what else, a level function, indicated by some notation like ' $\ell$ '. Level is also defined over a field of sets, but our inspiration comes not from point sets this time, but rather from formula sets. What we mean by a field of formula sets is the collection of all sets of formulas of some language $\mathcal{L}$, which we will call $\mathcal{P}(\mathbb{F})$-the power set of the set of formulas $\mathbb{F}$. Relative to certain systems of inference, there are properties which a given set of formulas might enjoy or not. Properties of that kind were used to define the concept of level in the first instance (see [4]). Such properties will serve us as useful examples in the present essay, but we shall take level functions to be defined more generally in terms of abstract properties of sets of formulas.

The original property of interest to the "preservationist" program of paraconsistent logic was classical consistency, indicated by $\mathrm{CON}_{\vdash}$ (where $\vdash$ is classical provability). What the level function does in that case is measure the level of inconsistency of the set of sentences in a sense that will be made clear. But before we get to level, we must take a detour through the concept of a cover.

2.1 A tale of two covers We consider two concepts of cover: one-the more common one, topological, and one-of our own devising, specific to logic. The general topological conception is a family of sets $\widetilde{\jmath}_{\xi}=\left\{\Delta_{i}: 1 \leq i \leq \xi\right\}$ such that for every $\beta \leq \xi, \mathcal{Q}\left(\Delta_{\beta}\right)$ and $\Gamma \subseteq \bigcup \mathfrak{\mho} \xi$, where $\mathbb{Q}$ is some property of formula sets. The $\xi$ subscript here is to say that $\widetilde{F}$ is a family over the ordinal $\xi$. The $\mathfrak{\wp} \xi$ is called a (Q)-cover. If $\Gamma=\bigcup \mathfrak{F}$ and for each $\Delta_{i}, \Delta_{j} \in \mathfrak{F}, i \neq j, \Delta_{i} \cap \Delta_{j}=\varnothing$, then we say that $\mathfrak{\wp}$ partitions $\Gamma$.

The notion of a logical cover, on the other hand, is one of a family of sets, indexed as before, although perhaps with a distinguished element (the earliest typically) which appears in every such family. ${ }^{2}$ Once again each element of the family must have some property $\mathcal{Q}$, but rather than the covered set being a subset of the union of the family, we require that the covered set be included in the union of the deductive closures (relative to some specified inference relation) of the elements of the cover.

We can bring the two notions into conformity by appeal to the topological notion of an open set. When the points on which the topology is constructed form a lattice, then what corresponds to the usual notion of openness is, ironically, virtually the same as deductive closure under the appropriate inference relation. With this in mind, a logical cover becomes a species of open cover which is the very meat and drink of topology. 
We refer to the deductive closure of a set $\Gamma$ relative to a logic $X$ as $\mathbb{C}_{X}(\Gamma)$. The $\mathcal{Q}$ which is important in the context of logical cover will be the consistency predicate of the logic $X, \operatorname{CON}_{X}$. Thus we can give our logic relevant definition of cover.

Definition 2.2 A logical cover $\mathfrak{F}=\left\{\Delta_{0}, \ldots, \Delta_{\xi}\right\}$ of $\Gamma$, where $\xi$ is as before, is such that

1. $\operatorname{CON}_{X}\left(\Delta_{i}\right)$ for each $i \leq \xi$,

2. $\Delta_{0}=\varnothing$, and

3. $\Gamma \subseteq \bigcup_{i \leq \xi} \mathbb{C}_{X}\left(\Delta_{i}\right)$.

Thus, for each $\psi \in \Gamma$ there is some $\Delta_{i}$ such that $\Delta_{i} \vdash_{X} \psi$. If the logic in question has the structural rule of inference [R], that is, if $\psi \in \Gamma$ then $\Gamma \vdash_{X} \psi$, then a partition of $\Gamma$ may serve as a logical cover (this will be of use later).

2.2 The road to level To define level we first define a predicate which holds between sets and ordinals. In relation to a property $\mathcal{Q}$ we define $\operatorname{COV}_{\mathcal{Q}}(\Gamma, \xi)$ if and only if there exists an $\mathfrak{\wp}$ which is a $\mathcal{Q}$-cover of $\Gamma$ and $\left|\widetilde{\jmath}-\left\{\Delta_{0}\right\}\right|=\xi$. Each $\Delta_{i}$ is referred to as a 'cell'. The $\xi$ is referred to as the 'width' of the cover. ${ }^{3}$ Now we can define level.

Definition 2.3 (General Level Function) A level function $\ell$ is an ordinal or $\{\infty\}$ valued function that "measures" the level of $\mathcal{Q}$-ness of a set of formulas $\Gamma$ where $\mathcal{Q}$ is a predicate of sets of formulas. The function is defined as

$$
\ell(\Gamma)= \begin{cases}\min \left\{\xi \mid \operatorname{COV}_{Q}(\Gamma, \xi)\right\} & \text { if it exists, } \\ \infty & \text { othewise. }\end{cases}
$$

The value of $\ell$ is the minimum value of the widths of the $\mathcal{Q}$-covers. The definition of the level function can be used with respect to either definition of cover. However, there is a rationale for choosing one definition of cover over the other. We want to keep the convention that the empty set has measure, or rather level, 0. But there are nonempty sets of measure 0 which have special properties and we would like to maintain that.

Suppose that $\Gamma$ is a set of classical tautologies. Such sets as $\Gamma$ are consistent but in a special way. Given any other classically consistent set $\Delta$, we know that $\Delta \cup \Gamma$ is also consistent. A general consistent set does not have this property. It is easy to see that the level of $\Delta \cup \Gamma$ is just the level of $\Delta$. If we use logical covers, for any $\Gamma \subseteq \mathbb{C}_{X}(\varnothing), \widetilde{F}=\{\varnothing\}$ (the cover consisting of just the empty set) is a cover of $\Gamma$ and $|\widetilde{\wp}-\{\varnothing\}|=0$. So sets of $X$-tautologies and the empty set have level 0 . The definition of logical cover allows nonempty sets of "measure" (i.e., level) 0! The topological cover, on the other hand, only allows $\varnothing$ to have level 0 since the union of the cells must contain the set being covered.

\section{Level Functions}

The restrictions which we impose on $\mathcal{Q}$ are applicable to either definition of cover. We require the property $\mathcal{Q}$ to be two things:

1. $\mathcal{Q}$ must be downward monotonic; that is, if $\Gamma \subseteq \Gamma^{\prime}$ and $\mathcal{Q}\left(\Gamma^{\prime}\right)$, then $\mathcal{Q}(\Gamma)$, and

2. the extension of $\mathcal{Q}$ must be nonempty.

The second requirement is trivial, but the first is to ensure the downward monotonicity of the level function relative to $\mathcal{Q}$. 
Proposition 3.1 If $\mathcal{Q}$ is downward monotonic, then $\ell$ is monotonic; that is, if $\Gamma \subseteq \Gamma^{\prime}$, then $\ell(\Gamma) \leq \ell\left(\Gamma^{\prime}\right)$.

Proof Suppose there were a subset with a larger level than the whole set. If there were a cover of the whole set of size, say $\xi$, then there would be a cover of the subset of size $\xi$ by the downward monotonicity of $\mathcal{Q}$, which contradicts the assumption that the subset has a larger level.

We shall assume in the sequel that $\mathcal{Q}$ is a downward monotonic property of sets of formulas; so level functions are monotonic.

What are the further properties of measure functions that should be preserved? Level functions are defined over the $\sigma$-algebra of the power set of formulas. The empty set has level 0 and there are nonempty sets of level 0 in certain contexts. Next we would like to see if level functions are countably additive. Level is not countably additive in general. One can see that it is not countably additive by considering the case of classical consistency. Assume the set $\Gamma=\{\psi, \varphi\}$ is consistent; but neither formula is a tautology and they are not equivalent. Then the unit sets $\{\varphi\}$ and $\{\psi\}$ are both subsets of $\Gamma$ and disjoint. These sets have level 1 since they can be covered by $\{\varnothing,\{\psi\}\}$ and $\{\varnothing,\{\varphi\}\}$, respectively, but neither by $\{\varnothing\}$ alone. Then each subset of $\Gamma$ is also consistent. But $1=\ell(\Gamma)=\ell(\{\varphi\} \cup\{\psi\}) \neq \ell(\{\varphi\})+\ell(\{\psi\})=2$.

The next phase is to prove a compactness theorem for level. First we must consider what compactness means in the context of level. In terms of consistency, compactness means $\Gamma$ is consistent if and only if every finite subset of $\Gamma$ is consistent. Thinking of level as a generalization of consistency, we say the level of the whole set is less than or equal to a certain number if and only if each finite subset's level is also less than or equal to that number. However, as we shall show, we can only make sense of this when two things obtain: the level is finite and $\mathcal{Q}$ is compact.

Let us first distinguish the case where $\ell(\Gamma)=\omega$ and $\ell(\Gamma)=\infty$. To say $\ell(\Gamma)=\omega$ means the width of the "smallest" possible $(\mathcal{Q})$-covers of $\Gamma$ is $\omega$. This does not mean that $\ell(\Gamma)=\infty$; that level is reserved for certain types of sets. For example, selfinconsistent sets like $\{\psi \wedge \neg \psi\}$, where $\mathcal{Q}$ is classical consistency, have level $\infty$. In the general case $\ell(\Gamma)=\infty$ means there are no $\mathcal{Q}$-covers of $\Gamma$. There being no $\mathcal{Q}$-covers just means that at least one of the unit subsets is not a $\mathcal{Q}$ set.

Classical logic provides an example of a set with level $\omega$. Consider, for example, $\left\{\left(\bigwedge_{i<n}\right) \wedge P_{n} \mid n \in \omega\right\}$, where each $P_{n}$ is an atomic sentence. This set has $\omega$ unit sets, each inconsistent with any other unit set, but each unit set is consistent. Thus, the minimal width of a cover is $\omega$.

Now we can explain why the level must be finite. Suppose we want 'level compactness' to mean 'if $\ell(\Gamma)=\xi$ then there is a finite subset of $\Gamma$ with level $\xi$ '. If this is our intent we will have a problem if the set in question has level $\omega$. Any finite set, which does not have level $\infty$, must have a finite level; since all of the unit sets are $\mathcal{Q}$-sets, the largest its level could be is the cardinality of the set. Thus, if a set $\Gamma$ has level $\omega$, no finite subset of $\Gamma$ can have level $\omega$. Therefore, our attempt at a description of level compactness at the beginning of the paragraph cannot succeed for infinite levels (and we want it to succeed).

The reason we want level compactness to be characterized by 'if $\ell(\Gamma)=n$ then there is a finite subset of $\Gamma$ with level $n$ ' is because we want it to be equivalent to $' \ell(\Gamma) \leq n \Longleftrightarrow \forall \Gamma^{\prime} \subseteq \Gamma$ that are finite, $\ell\left(\Gamma^{\prime}\right) \leq n$ for all $n \in \omega^{\prime}$. This can only occur if the level is finite (as we saw above) and when $\mathcal{Q}$ is compact. Thus we assert 
(1) $\mathcal{Q}$ must be compact and (2) we must restrict our application of level compactness to the finite values of the level function. So the level function is only compact on a subset of $\mathcal{P}(\mathbb{F})$.

Using finite levels we can make the following assumption about level.

Proposition 3.2 If $m \in \omega, \ell(\Sigma)=m<\ell(\Sigma \cup \Gamma)$, and $\mathcal{Q}(\Gamma)$, then $\ell(\Gamma \cup \Sigma)=m+1$.

Proof If $m=\ell(\Sigma)<\ell(\Sigma \cup \Gamma)$, and $\mathcal{Q}(\Gamma)$ then suppose $\widetilde{\mho}_{m}=\left\{\Delta_{0}, \Delta_{1}, \ldots, \Delta_{m}\right\}$ is a minimal cover for $\Sigma$. The cover $\widetilde{F}_{m}^{\prime}=\left\{\Delta_{0}, \Delta_{1}, \ldots, \Delta_{m}, \Gamma\right\}$ will be a minimal cover for $\Sigma \cup \Gamma$ of width $m+1$. Hence $\ell(\Sigma \cup \Gamma)=m+1$.

Thus, one may assume, without loss of generality, that the set being added is consistent because if the level goes up at all, shy of $\infty$, it must go up by at least 1 , and there will always be a $\mathcal{Q}$-set contained in the set being added. Not only does the above proposition hold, but if $\Gamma$ is a 0 set and $\Pi$ has some finite level, then $\Gamma \cup \Pi$ will have the level of $\Pi$. This is left as an exercise. Now we will show our restrictions to be worthwhile since with them the previously mentioned descriptions of level compactness are equivalent.

\section{Level Compactness}

Theorem 4.1 (d'Entremont [2], Theorem 10) The following are equivalent when $\ell(\Gamma)<\omega$ and $\mathcal{Q}$ is compact:

1. $\ell(\Gamma) \leq n \Longleftrightarrow \forall \Gamma^{\prime} \subseteq \Gamma$ which are finite, $\ell\left(\Gamma^{\prime}\right) \leq n$;

2. if $\ell(\Gamma)=n$, then $\exists \Gamma^{\prime} \subseteq \Gamma$ which is finite, such that $\ell\left(\Gamma^{\prime}\right)=n$;

3. if there is a finite subset $\Gamma^{*}$ of $\Gamma$ such that for any other finite $\Gamma^{\prime} \subseteq \Gamma$, $\ell\left(\Gamma^{\prime}\right) \leq \ell\left(\Gamma^{*}\right)$, then $\ell\left(\Gamma^{*}\right)=\ell(\Gamma)$.

Proof We proceed by showing the equivalence in a triangle. All $n, m, k$, and so on, are elements of $\omega$.

$1 \Rightarrow 2$ Assume (1) and assume for reductio that $\ell(\Gamma)=n$ and that there is no finite subset of $\Gamma$ which has level $n$. We know by monotonicity of level that all of the subsets of $\Gamma$ must have level less than that of $\Gamma$, so there is an upper bound. This upper bound will also apply to finite sets. Call this upper bound $m$. This $m$ is strictly less than $n$ because otherwise there would be a finite subset of level $n$ which there isn't. With (1) we get $\ell(\Gamma) \leq m<n$, which is a contradiction.

$2 \Rightarrow 3$ Assume (2) and the existence of a $\Gamma^{*}$ as in the antecedent of (3). There must be, by (2), a finite $\Gamma^{\prime} \subseteq \Gamma$ with $\ell\left(\Gamma^{\prime}\right)=\ell(\Gamma)$, but then $\ell(\Gamma)=\ell\left(\Gamma^{\prime}\right) \leq \ell\left(\Gamma^{*}\right)$. By monotonicity of $\ell$ we get $\ell\left(\Gamma^{*}\right) \leq \ell(\Gamma)$; hence, $\ell\left(\Gamma^{*}\right)=\ell(\Gamma)$.

$3 \Rightarrow 1$ Assume (3). The only if direction of (1) follows from monotonicity of $\ell$; thus, assume that for every finite $\Gamma^{\prime} \subseteq \Gamma \ell\left(\Gamma^{\prime}\right) \leq n$. Let $m=\max \left\{k \mid \ell\left(\Gamma^{\prime}\right)=k \& \Gamma^{\prime}\right.$ $\subseteq \Gamma$ finite $\}$. This must exist since there is an upper bound, namely, $n$. Let $\Gamma^{*}=\Gamma^{\prime}$ such that $\ell\left(\Gamma^{\prime}\right)=m$. Then we have satisfied the conditions for (3); thus, $\ell\left(\Gamma^{*}\right)=\ell(\Gamma)=m \leq n$.

Using these equivalences we can actually prove that, for finite level, the level function is compact in the way mentioned as equivalence (1) in Theorem 4.1. In the case of logical covers we will make the assumption that the consistency of $\Gamma$ means 
that $\mathbb{C}_{X}(\Gamma) \neq \mathbb{F}$. The compactness theorem holds for either notion of cover we use, topological or logical. ${ }^{4}$

Theorem 4.2 (d'Entremont, Theorem 9 [2], Finite Level Compactness) If $\Gamma$ is a set of formulas with $\ell(\Gamma)<\omega$ and $Q$ is compact, then $\ell(\Gamma) \leq n$ if and only if for every finite subset $\Gamma^{\prime}$ of $\Gamma, \ell\left(\Gamma^{\prime}\right) \leq n$.

Proof Theorem 4.1 says that the following are equivalent for all $n \in \omega$. (We will use these at various stages.)

1. $\ell(\Gamma) \leq n \Longleftrightarrow \forall \Gamma^{\prime} \subseteq \Gamma$ which are finite, $\ell\left(\Gamma^{\prime}\right) \leq n$.

2. If $\ell(\Gamma)=n$ then $\exists \Gamma^{\prime} \subseteq \Gamma$ which is finite, such that $\ell\left(\Gamma^{\prime}\right)=n$.

3. When $\ell(\Gamma)=n$, if there is a finite subset $\Gamma^{*}$ of $\Gamma$ such that, for any other finite $\Gamma^{\prime} \subseteq \Gamma, \ell\left(\Gamma^{\prime}\right) \leq \ell\left(\Gamma^{*}\right)$, then $\ell\left(\Gamma^{*}\right)=\ell(\Gamma)$.

We will proceed by induction on the level of $\Gamma$. For $\ell(\Gamma)=0$, we know that $\Gamma=\varnothing$ or $\Gamma \subset \mathbb{C}_{Q}(\varnothing),{ }^{5}$ so $\varnothing \subseteq \Gamma$ and is finite with $\ell(\varnothing)=0$, and by the monotonicity of $\mathcal{Q}$, any nonempty subset of a nonempty $\Gamma$ will have level 0 . Thus we get the result for the basis step by showing (2). Assume that $\ell(\Gamma) \leq k$ if and only if, for every finite subset $\Gamma^{\prime}$ of $\Gamma, \ell\left(\Gamma^{\prime}\right) \leq k$ for $k \leq n$. Assume that $\ell(\Gamma)=n+1$. We will show that (3) holds to get the result for this stage. Assume as in the antecedent of (3) that there is a finite $\Gamma^{*} \subseteq \Gamma$ and for any finite $\Gamma^{\prime} \subseteq \Gamma, \ell\left(\Gamma^{\prime}\right) \leq \ell\left(\Gamma^{*}\right)$. Clearly, $\ell\left(\Gamma^{*}\right) \leq \ell(\Gamma)$. If $\ell\left(\Gamma^{*}\right)=\ell(\Gamma)$ then we are done. So assume $\ell\left(\Gamma^{*}\right)<\ell(\Gamma)=n+1$. Thus, $\ell\left(\Gamma^{*}\right) \leq n$. This means that every finite subset of $\Gamma$ has level $\leq n$, but by inductive hypothesis, and using (1), we get that $\ell(\Gamma) \leq n<n+1=\ell(\Gamma)$. But that is a contradiction. Hence, $\ell\left(\Gamma^{*}\right)=\ell(\Gamma)$, which is what we wanted. So we have shown (3) for $\ell(\Gamma)=n+1$, which is equivalent to $\ell(\Gamma) \leq n+1$ if and only if, for every finite subset $\Gamma^{\prime}$ of $\Gamma, \ell\left(\Gamma^{\prime}\right) \leq n+1$. Therefore, for all $n \in \omega, \ell(\Gamma) \leq n$ if and only if, for every finite subset $\Gamma^{\prime}$ of $\Gamma, \ell\left(\Gamma^{\prime}\right) \leq n$.

The compactness of the predicate $\mathcal{Q}$ will carry over to make the level function defined on it compact. Thus, we can look at some applications of the finite level compactness theorem.

\section{Applications}

These concepts were developed in the context of logic, so of course they find their application there. From here on let $X$ be some logic over a countable language $\mathcal{L}$. Then we have the logic's syntactic consistency predicate and inference relation as was defined earlier. We take the extensional meaning of 'logic' so that a logic is the set of pairs $\langle\Gamma, \psi\rangle$ such that $\Gamma \vdash_{X} \psi$. Taking the syntactic consistency predicate of $X$ as $\mathcal{Q}$ we can force $\mathcal{Q}$ to be compact since it is common to consider proofs finite. Concerning $X$ we will allow $\vdash_{X}$ to be reflexive, monotonic, and transitive, that is, allow the rules $[\mathrm{R}],[\mathrm{M}]$, and $[\mathrm{T}]$ a la Scott $[6]$. As a corollary to the compactness theorem we can get an extension lemma. For this we need a definition.

Definition 5.1 $\quad \Gamma^{+}$is a maximal level preserving extension (MLPE) of $\Gamma$ if and only if

1. $\Gamma \subseteq \Gamma^{+}$,

2. $\ell\left(\Gamma^{+}\right)=\ell(\Gamma)$, and

3. for any formula $\psi$, if $\ell\left(\Gamma^{+} \cup\{\psi\}\right)=\ell\left(\Gamma^{+}\right)$, then $\psi \in \Gamma^{+}$. 
The "absolute" definition, one not relative to any $\Gamma$, just satisfies the third clause. Such sets are maximal with respect to level; that is, you can't add anything to them without raising the level. As one would expect we can produce one of these $\Gamma^{+}$ sets by the following construction. Recall that the language that we are using is countable.

Lemma 5.2 (Lindenbaum Level Lemma) Let $\Gamma$ have level $m \in \omega$; then it can be extended to a $\Gamma^{+}$.

Proof Let $\Gamma$ have level $m \in \omega$. Then let $\psi_{0}, \psi_{1}, \ldots, \psi_{k}, \ldots$ be such that $k \in \omega$ is an enumeration of the formulas of the language. Form sets $\Sigma_{n}$ for $n \in \omega$ by

$$
\begin{gathered}
\Sigma_{0}=\Gamma \\
\Sigma_{n}= \begin{cases}\Sigma_{n-1} \cup\left\{\psi_{n}\right\} & \text { if } \ell\left(\Sigma_{n-1} \cup\left\{\psi_{n}\right\}\right)=\ell(\Gamma), \\
\Sigma_{n-1} & \text { otherwise. }\end{cases}
\end{gathered}
$$

Let $\Gamma^{+}=\bigcup_{n \in \omega} \Sigma_{n}$.

Claim This set is a level preserving maximal extension of $\Gamma$. By the recursive construction and compactness $\ell\left(\Gamma^{+}\right)=\ell(\Gamma)$. There are three cases:

1. $\ell\left(\Gamma^{+}\right)=\infty$.

2. $\ell\left(\Gamma^{+}\right) \geq \omega$.

3. $\omega>\ell\left(\Gamma^{+}\right)>\ell(\Gamma)$.

If (1), then a formula was added which cannot be covered, but that is impossible. If (2), then for any $n \in \omega$ there is a finite subset of $\Gamma^{+}$of level $n$. If there were a finite upper bound on the levels of the finite subsets of $\Gamma^{+}$, then the compactness theorem says the whole set would have that finite level, which is contrary to assumption. So choose $n>\ell(\Gamma)$; the finite subset of level $n$ will be contained in some $\Sigma_{k}$ so $\ell\left(\Sigma_{k}\right)>\ell(\Gamma)$ which is impossible. Finally, if (3), there was a finite set which was the culprit, and it would be contained in some $\Sigma_{n}$, which is also impossible. Thus $\ell\left(\Gamma^{+}\right)=\ell(\Gamma)$.

Lastly, suppose $\ell\left(\Gamma^{+} \cup\{\varphi\}\right)=\ell\left(\Gamma^{+}\right)$; then either (a) $\varphi \in \Gamma$ or, if not, (b) $\varphi=\psi_{n}$ for some $n \in \omega$. If (a) then $\varphi \in \Gamma^{+}$a fortiori. If (b) then $\varphi$ was considered for membership at stage $n$, and since adding it to $\Gamma^{+}$does not change its level, adding $\varphi$ to $\Sigma_{n-1}$ does not change the level of $\Sigma_{n-1}$ since $\ell\left(\Sigma_{n}\right)=\ell(\Gamma)=\ell\left(\Gamma^{+}\right)$. Thus, $\varphi$ was added at stage $n$. Therefore, $\varphi \in \Gamma^{+}$.

So we can always extend sets with finite level to an MLPE.

To make level applicable to inference we consider the general concept of "Forcing." Given a logic $X$ we can define a forcing relation $\left(\left[\|_{X}\right)\right.$ on the provability predicate $\vdash_{X}$. We do this using the definition of logical cover and level where $\mathcal{Q}$ is $\operatorname{CON}_{X}$.

Definition 5.3 $\Gamma X$-Forces $\psi, \Gamma\left[\Vdash_{X} \psi\right.$ if and only if every logical cover of $\Gamma$ of width $\ell_{X}(\Gamma)$ contains a cell, $\Delta_{i} \in \mathfrak{F}$, such that $\Delta_{i} \vdash_{X} \psi$.

So we can rephrase this to say $\Gamma \mathrm{X}$-Forces $\psi$ if and only if all the 'narrowest' covers contain a cell which $X$-proves $\psi$. We will abbreviate the class of narrow covers of $\Gamma$ as $\operatorname{NAR}(\Gamma)$. The level function $\ell_{X}$ defined with respect to $\operatorname{CON}_{X}$ is implicit.

An important feature of forcing is that it is an inference relation which preserves $X$-level. The following result proves this. 
Lemma 5.4 $\ell_{X}\left(\mathbb{C}_{\left[\vdash_{X}\right.}(\Gamma)\right)=\ell_{X}(\Gamma)$; that is, the $X$-Forcing closure of $\Gamma$ has the same level as $\Gamma$.

From here on we will omit the subscripts in the proofs, since we take it to be understood which $X$ we are working with.

Proof Assume $\ell(\Gamma)=n$ finite. So then $\psi \in \mathbb{C}_{[\Vdash \Vdash}(\Gamma)$ if and only if $\Gamma[\Vdash \psi$. Let $\mathfrak{\Im} \in N A R(\Gamma)$. Then, by definition of forcing, for each $\psi \in \mathbb{C}_{[\Vdash T}(\Gamma)$, there is $\Delta_{i} \in \mathfrak{\wp}$ such that $\Delta_{i} \vdash \psi$. Thus, $\mathfrak{\wp}$ is a cover of $\mathbb{C}_{[\Vdash}(\Gamma)$. Further, it is easy to see that $[\Vdash$ has $[\mathrm{R}]$, so $\Gamma \subseteq \mathbb{C}_{[\Vdash}(\Gamma)$. So by monotonicity of level $\ell(\Gamma) \leq \ell\left(\mathbb{C}_{[\Vdash}(\Gamma)\right)$, but $\mathfrak{\wp}$ is a cover of $\mathbb{C}_{[\Vdash}(\Gamma)$ of width $\ell(\Gamma)$; therefore, $\ell_{X}\left(\mathbb{C}_{\left[{ }_{X}\right.}(\Gamma)\right)=\ell_{X}(\Gamma)$.

As a corollary to this lemma we have the following.

Corollary 5.5 If $\Gamma\left[\Vdash_{X} \psi\right.$, then $\ell_{X}(\Gamma \cup\{\psi\})=\ell_{X}(\Gamma)$.

One can see in this context that level is a generalization of consistency. The first two levels, 0 and 1 , use $\operatorname{coN}_{X}$ but 'after' that, to say that a set $\Gamma$ is inconsistent with another set $\Pi$ is to say that $\ell(\Gamma)<\ell(\Gamma \cup \Pi)$. To say that a set is universally inconsistent is to say that the set has level $\infty$. And forcing is a relation which preserves this new type of consistency.

Our goal is to derive something like a maximal provability theorem like we have for classical logic. We want that a set $\Gamma$ forces a formula if and only if every 'maximal extension' of $\Gamma$ forces the formula. The maximal extensions in our case are the MLPES. One can see from Corollary 5.5 that MLPES only force those formulas which they contain; so they are clearly a generalization of classical maximally consistent sets. However, to do this we need to impose a bit of structure on the logic in question. We must consider a certain class of logics with some special properties.

Recall the discussion of self inconsistent formulas or unit sets with level $\infty$. These 'absurd' formulas make an appearance in our next definition.

Definition 5.6 (Denial) A logic $X$ has Nontrivial Denial if and only if for each nonabsurd formula $\psi$ there is another nonabsurd formula $\psi^{\prime}$ - such that $\overline{\operatorname{CON}_{X}}\left(\left\{\psi, \psi^{\prime}\right\}\right)$, where the overline indicates predicate negation.

The two formulas in this definition are said to deny each other. Without the restriction to nonabsurd formulas, the above defines what it is for a logic $X$ to have denial simplicater.

We require that denial commutes in the right way with consistency and $\vdash_{X}$. This is to say that we impose the condition

[Den] $\quad \Gamma \vdash_{X} \psi \Longleftrightarrow \overline{\operatorname{CON}_{X}}\left(\left\{\Gamma, \psi^{\prime}\right\}\right)$ where $\psi^{\prime}$ denies $\psi$.

There does not have to be a unique denial nor is denial necessarily functional in the way that classical negation is. However, denial must be symmetric, so $\psi$ denies $\varphi$ if and only if $\varphi$ denies $\psi$. We need a much stronger property to get to our final destination. This property is shared by many logics such as intuitionistic logic, quantum logic, and so forth. We call logics with this property 'productival'.

Definition 5.7 A logic $X$ is productival if and only if, given any finite set $\Gamma$, there is a formula $\varphi$ such that $\varphi \vdash_{X} \psi$ for each $\psi \in \Gamma$, and $\Gamma \vdash_{X} \varphi$.

We can now use our extension lemma to prove a maximal forcibility theorem for the class of logics which satisfy the two properties above. 
Theorem 5.8 (Maximal Forcibility Theorem) Let $X$ be a productival logic which has denial and $\ell(\Gamma) \in \omega$. Then $\Gamma\left[\Vdash_{X} \psi\right.$ if and only if, for each MLPE $\Gamma^{+}$of $\Gamma$, $\Gamma^{+}\left[\Vdash_{X} \psi\right.$.

Proof (only if) Suppose $\Gamma\left[\Vdash \psi\right.$. Then let $\Gamma^{+}$be an MLPE of $\Gamma$. So, $\ell(\Gamma)=\ell\left(\Gamma^{+}\right)$ by definition. Suppose that $\widetilde{\mathfrak{r}} \in N A R\left(\Gamma^{+}\right)$; then $\widetilde{\wp}$ is also a cover of $\Gamma$ because $\Gamma \subseteq \Gamma^{+}$. But the width of $\widetilde{\mho}$ is also the level of $\Gamma$, so $\mathfrak{\mho} \in N A R(\Gamma)$. Thus by definition of Forcing there must be $\Delta_{i} \in \mathfrak{F}$ such that $\Delta_{i} \vdash \psi$. Since $\mathfrak{\wp}$ was arbitrary, $\Gamma^{+}[\Vdash \psi$.

(if) By contrapositive. Assume $\Gamma[\| \psi$ and $\ell(\Gamma)=m$. Then there is an $\mathfrak{\Im} \in \operatorname{NAR}(\Gamma)$ which is a partition of $\Gamma$ where no $\Delta_{i} \in \mathfrak{F}$ is such that $\Delta_{i} \vdash \psi$. Thus, by [Den] $\operatorname{CoN}\left(\Delta_{i} \cup\left\{\psi^{\prime}\right\}\right)$ for each $i, \psi^{\prime}$ a denial of $\psi$. For each $\Delta_{i} \neq \Delta_{j}$, $\Delta_{i} \cap \Delta_{j}=\varnothing$. And $\overline{\operatorname{CON}}\left(\Delta_{i} \cup \Delta_{j}\right)$. By compactness of CON we have for each pair of cells finite sets $\Delta_{i}^{\prime}$ and $\Delta_{j}^{\prime}$, contained in $\Delta_{i}, \Delta_{j}$, respectively, which are inconsistent with one another. Thus we form their respective products and get $\varphi_{i j}$ and $\varphi_{j i}$. So we have $\overline{\mathrm{CON}}\left(\left\{\varphi_{i j}\right\} \cup\left\{\varphi_{j i}\right\}\right)$. Since there are only finitely many of these $\varphi_{i j}$ s for each $i$ we can get a product for each $i$, call it $\varphi_{i}$, such that $\varphi_{i} \vdash \varphi_{i j}$ for each $j \neq i$. The $\varphi_{i} \mathrm{~s}$ are clearly consistent with the $\Delta_{i}$ s and any two distinct $\varphi_{i}$ s are inconsistent. Since $\operatorname{CON}\left(\Delta_{i} \cup\left\{\psi^{\prime}\right\}\right)$ we can form the product of $\left\{\varphi_{i}, \psi^{\prime}\right\}$ to get $\varphi_{i}^{*}$ for each $i$, which will also be consistent with each $\Delta_{i}$. Form the cover,

$$
\widetilde{\mho}^{\prime}=\left\{\varnothing, \Delta_{i} \cup\left\{\varphi_{i}^{*}\right\}: \Delta_{i} \in \widetilde{\mho}, \& 1 \leq i \leq m\right\} .
$$

$\widetilde{\mho}^{\prime}$ is a cover of $\Gamma \cup\left\{\psi^{\prime}\right\}$ of width $m$. By monotonicity of level we get that $\ell\left(\Gamma^{*}\right)=\ell\left(\Gamma \cup\left\{\varphi_{i}^{*}: 1 \leq i \leq m\right\}\right)=\ell(\Gamma)$. Extend this new set $\Gamma^{*}$ to a $\Gamma^{+}$as in the extension lemma. This $\Gamma^{+}$will have level $m$ and since each $\varphi_{i}^{*}$ proves $\psi^{\prime}$ and must be contained in a different cell, $\psi$ cannot be added to $\Gamma^{+}$without increasing its level, which is to say that $\Gamma^{+}\left[\mid \psi \psi\right.$ with $\Gamma \subseteq \Gamma^{+}$.

Where to go from here? It would be nice to know where the $X$-Forcing relation fits with respect to other inference relations that preserve $X$-level.

As we mentioned above, all of the logics $X$ that we consider have [R], [T], and $[\mathrm{M}]$. However, forcing does not have [M] but a variation of it. It is well known in the literature ${ }^{6}$ that classical forcing is monotonic in a restricted sense. The 'restricted sense' is as follows: if $\Gamma[\Vdash \varphi$ and $\ell(\Gamma \cup \Delta)=\ell(\Gamma)$, then $\Gamma \cup \Delta[\Vdash \varphi$. Thus, if a set can be added without changing the level, then the forcing consequences are preserved by the union. We will call this restricted sense of monotonicity [LM]. (Level-Monotonicity, and relative to $X, X$-[LM].) The generalization from classical[LM] to $X$-[LM] follows.

Proposition 5.9 If $\Gamma\left[\Vdash_{X} \varphi, \Gamma\right.$ has finite level and $\ell_{X}(\Gamma)=\ell_{X}(\Gamma \cup \Delta)$, then $\Gamma \cup \Delta\left[\Vdash_{X} \varphi\right.$.

Proof This is a corollary to the first direction in Theorem 5.8 since any level preserving extension will be contained in some MLPE. So, a fortiori, $\Gamma \cup \Delta[\Vdash \varphi$.

Since we wanted the "base" logics such as $X$ to have [R], [T], and [M] we will demand that inference relations which preserve $X$-level must have [R], [T], and $X$ [LM]. These restrictions give enough information to see where $X$-forcing fits into the picture. 
Theorem 5.10 (Level Preservation) If $X$ is a logic as in Theorem 5.8 and $Y$ is another logic which preserves $X$-level, obeys [R], [T], and is monotonic with respect to X-level preserving extensions, then for all $\Gamma, \mathbb{C}_{Y}(\Gamma) \subseteq \mathbb{C}_{[\Vdash X}(\Gamma)$. Thus, X-Forcing is the largest relation which preserves $X$-level and has $X$-[LM].

This is not always the case when $X$ is not as in Theorem 5.8. The biggest relation which is like forcing is defined by

$$
\Gamma \vdash_{\mathrm{MLPE}} \varphi \Leftrightarrow \varphi \in \bigcap_{\Gamma^{+} \in \mathrm{MLPE}} \Gamma^{+}
$$

where MLPE stands for the class of maximal level preserving extensions of $\Gamma$. We abbreviate the right-hand side as $\bigcap \Gamma^{+}$.

Proposition 5.11 Given a logic $X$ and a relation $Y$ like the relation $Y$ in Theorem 5.10, then, for all $\Gamma, \mathbb{C}_{Y}(\Gamma) \subseteq \bigcap \Gamma^{+}$.

Proof Assume for reductio that $\varphi \in \mathbb{C}_{Y}(\Gamma)$ but $\varphi \notin \bigcap \Gamma^{+}$. Then there is an MLPE of $\Gamma$, call it $\Gamma_{\varphi}^{+}$, such that $\varphi \notin \Gamma_{\varphi}^{+}$. By definition $\ell\left(\Gamma_{\varphi}^{+} \cup\{\varphi\}\right)>\ell\left(\Gamma_{\varphi}^{+}\right)=\ell(\Gamma)$. And since $\Gamma \subseteq \Gamma_{\varphi}^{+}$we have by hypothesis on $Y, \mathbb{C}_{Y}(\Gamma) \subseteq \mathbb{C}_{Y}\left(\Gamma_{\varphi}^{+}\right)$. Thus $\varphi \in \mathbb{C}_{Y}\left(\Gamma_{\varphi}^{+}\right)$ and so $\ell\left(\mathbb{C}_{Y}\left(\Gamma_{\varphi}^{+}\right)\right)>\ell\left(\Gamma_{\varphi}^{+}\right)$. Therefore $Y$ does not preserve $X$-level contrary to hypothesis.

Proof of Theorem 5.10 Given a logic $X$, and $Y$ is as required, we know that $\mathbb{C}_{Y}(\Gamma) \subseteq \bigcap \Gamma^{+}$. But Theorem 5.8 says that for productival logics $\mathbb{C}_{\left[{ }_{X}\right.}(\Gamma)=\bigcap \Gamma^{+}$. Hence, by Proposition 5.11, we get $\mathbb{C}_{Y}(\Gamma) \subseteq \mathbb{C}_{[{ } X}(\Gamma)$.

\section{Notes}

1. In measure theory of the unit interval the $\sigma$-algebra is some proper subset of $\mathcal{P}([0,1])$. Using the axiom of choice one can prove that not all subsets of [0,1] are, in fact, measurable. However, our generalization will not suffer this problem, with or without choice.

2. In the work of Brown and Schotch [1], the empty set serves as such an element.

3. However, $\xi$ can be transfinite, but in that case, $|\xi-1|=\xi$, which is what we want.

4. However, we provide the proof for the logical version since it is inclusive of the topological version. The only difference is in the basis step of the induction.

5. This assertion of a $\mathcal{Q}$-closure is for the case where we use logical covers and $\mathcal{Q}$ is $\operatorname{CON}_{X}$; to consider the topological covers we restrict the basis case to $\varnothing$ and just use $\mathcal{Q}$ and no closure operator.

6. See [5], p. 312 and [1], p. 275.

\section{References}

[1] Brown, B., and P. Schotch, "Logic and aggregation," Journal of Philosophical Logic, vol. 28 (1999), pp. 265-87. Zbl 0931.03041. MR 1699057. 554 
[2] d'Entremont, B., "Inference and Level," master's thesis, Dalhousie University, Halifax, 1982. 549,550

[3] Jao, D., “Measure,” Online Encyclopedia: Planetmath.org, 2006. 546

[4] Schotch, P. K., and R. E. Jennings, "Inference and necessity," Journal of Philosophical Logic, vol. 9 (1980), pp. 327-40. Zbl 0442.03020. MR 585758. 545, 546

[5] Schotch, P. K., and R. E. Jennings, "On detonating," pp. 306-27 in Paraconsistent Logic. Essays on the Inconsistent, edited by G. Priest, R. Routley, and J. Norman, Analytica, Philosophia Verlag GmbH, Munich, 1989. Zbl 0695.03012. MR 1077648. 545, 554

[6] Scott, D., "Completeness and axiomatizability in many-valued logic," pp. 411-35 in Proceedings of the Tarski Symposium (Proceedings of the Symposium in Pure Mathematics, Vol. XXV, Berkeley, 1971), American Mathematical Society, Providence, 1974. Zbl 0318.02021. MR 0363802. 550

\section{Acknowledgments}

To the memory of Catherine Bennett, for everything. The authors wish to acknowledge the support of SSHRCC under research grant 410-2005-1088 and the help of Dr. Peter Schotch for comments during the preparation of this paper.

Department of Philosophy

Dalhousie University

Halifax NS

CANADA B3H $4 \mathrm{P9}$

gpayette@dal.ca

dentremo@gov.ns.ca 